\title{
Spectral properties of reflective interference filters
}

\author{
V.Yu. Pervak, L.V. Poperenko \\ Taras Shevchenko Kiev University, \\ prosp. Acad.Glushkova 6, 01028 Kiev, Ukraine
}

\section{Yu.A. Pervak}

Institute of Semiconductor Physics, National Academy of Sciences of Ukraine, prosp. Nauki 45, 03650 Kiev, Ukraine. Phone (38044) 265 1817, Fax (38044) 2658342. E-mail:Pervak@dep48.semicond.kiev.ua

\begin{abstract}
The spectral properties of band reflective interference filters are investigated. The operation of the filter is based on the method of residual rays. Angular dependences of the filter transmission bandshape on the parameters of multilayer coating, such as the thickness, refractive indexes, the number of the layers, are analyzed.
\end{abstract}

Keywords: interference filters, suppression of background transmission.

Paper received 02.11.00; revised manuscript received 23.01.01; accepted for publication 16.02.01.

\section{Introduction}

Recently in [1] it was shown that the filter transmission can be increased up to $90 \%$ for the specified background transmission. In short, the essence of the task consists in the following. Any interference filter is known to be a certain multilayer system consisting of the consequently deposited layers made of materials with different refractive indices [2-6]. Most often, only two alternating layers from film-making materials are involved. In the case of narrow-band filters, the optical thickness of the layers is $\lambda_{0} / 4$, whereas that of a layer in the middle of the multilayer system (Fabry-Perot filters) [2] or of several non-adjacent layers (multihalf-wave filters) $[4,5]$ is multiple of $\lambda_{0} / 2$, where $\lambda_{0}$ is the wavelength of the peak transmission of the Fabry-Perot filter or, in the case of multihalf-wave filter, the wavelength of the transmission band (or bands) center. The cut-off shortwave and long-wave filters contain no half-wave layers, while the optical thickness of their outer layers made of the same material usually equals $\lambda_{0} / 8$, where $\lambda_{0}$ is the wavelength of the center of a high reflection band. To obtain the more smoothed spectral characteristics of the cut-off filters, the optical thickness of some layers is made different from the quater-wavelength one. The number and position of such layers are determined by various optimization procedures $[3,6]$. The disadvantage common to the multilayer systems (not only to the filters) is their comparatively narrow high- transmission and high-reflecrion bands. For example, in the case of two-component interference filters, the shortwavelength $\lambda_{S}$ and the long-wavelength $\lambda_{L}$ edges of the background suppression regions coincide with the edges of the first zone of high reflection from a multilayer stack formed by a succession of alternating layers made of materials with the high $\left(n_{H}\right)$ and low $\left(n_{L}\right)$ refractive indices and the optical thickness $\lambda_{0} / 4$. They are given by the well-known expression [3]:

$$
\begin{aligned}
& \lambda_{L, S}=\lambda_{0} \pm \Delta \lambda_{\mathrm{L}, S} ; \Delta \lambda_{L, S}=\lambda_{0} \Delta\left(\frac{\lambda_{0}}{\lambda}\right) /\left[1 \mp \Delta\left(\frac{\lambda_{0}}{\lambda}\right)\right] ; \\
& \Delta\left(\frac{\lambda_{0}}{\lambda}\right)=\frac{2}{\pi} \arcsin \left[\frac{n_{H}-n_{L}}{n_{H}+n_{L}}\right] .
\end{aligned}
$$

When the optical thickness deviates from the quaterwavelength one, while keeping the half-wavelength thickness of the repeated period, $\Delta \lambda$ decreases $[7,8]$.

The background suppression region of the interference filter is usually extended by adding an absorption filter or several interference systems with appropriate spectral parameters. Unfortunately, the proper additional absorption filters are not always available, espesially for the operating range of the filter spreading from UV to IR. The most difficult problem is producing UV interference filters; the reason for this being the almost complete 
lack of the materials simultaneously transparent in UV and absorbing in visible and near IR spectral regions. An increase in the thickness of the absorption filter aimed at increasing the coefficient of transmission suppression in the background spectral range results, at the same time, in the transmission drop at the operating wavelength. For this reason, the UV interference filters presently have the peak transmission at a level of $20-25 \%$.

To suppress the background transmission, in work [1] it was proposed to use reflective filters. The design of such reflective filter is shown in Fig. 1. It includes (a) two or (b) four plane-parallel plates placed parallel in pair. The surfaces facing each other are coated with multilayer dielectric mirrors. In work [1] investegated was a case when the multilayer coatings of plates are identical. Below we shall consider, how it is possible to change spectral characteristics of the filters, if one uses the cut-off filters as multilayer mirrors.

\section{Results and discussion}

That the suppression of a background radiation was identical till both legs from a passband of a reflective filter, on one plate the multilayer coating of the shortwave cut-off filter, and on another long wavelength is superimposed. The constructions of the cut-off filters were selected so that in the spectral region from 300 up to $2000 \mathrm{~nm}$ the plate with coatings was not absorbing. The construction of the cut-off filters was selected in such manner that at fixed optical constants of separate layers the width of a transmission band inherent to a reflective filter was maximum. Besides, with the purpose of deriving more uniform spectral dependence of the transmission of cut-off filters in their basic operation spectral range the standard construction of cut-off filters was supplemented by a system of matched layers. The shotwave cut-off filter consists of the fused silica substrate $S\left(n_{S}=1.45\right)$ and the multilayer system $(\mathrm{HLH})^{12}[0.92(\mathrm{HLH})]^{3}$ formed by the alternating layers with the high $\left[n_{H}=2.0\left(\mathrm{HfO}_{2}\right)\right]$ and low $\left[n_{L}=1.45\right.$ $\left.\left(\mathrm{SiO}_{2}\right)\right]$ refractive indices. The optical thicknesses of $H$-layers are equal to a half of quarter of the wavelength $\lambda_{0}$ and the optical thicknesses of $L$-layer are equal to a quarter of the wavelength $\lambda_{0}$, where $\lambda_{0}$ is some fixed wavelength, which determines a position of an edge of filter transmission. The basic system (HLH) ${ }^{12}$ is the the stan-

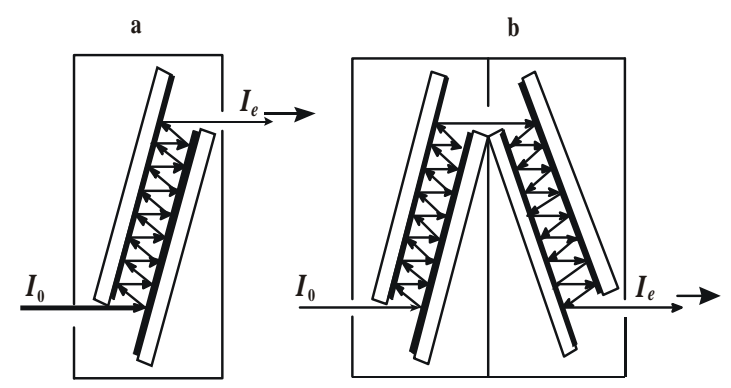

Fig. 1. Design of the reflective filter. dard construction of shotwave cut-off filters and the system $[0.92(\mathrm{HLH})]^{3}$ is the matched system, in which the optical thicknesses of layers differ from appropriate optical thicknesses of the basic system at 0.92 of time. Fig. 2 presents the spectral dependence of the reflection of the shotwave cut-off filter at the various angles of incidence $\theta$. The longwave cut-off filter consists of the fused silica substrate $S\left(n_{S}=1.45\right)$ and the multilayer system $(\mathrm{LHL})^{12}[1.08(\mathrm{LHL})]^{3}$ formed by the alternating layers with the high $\left[n_{H}=2.0\left(\mathrm{HfO}_{2}\right)\right]$ and low $\left[n_{L}=1.45\left(\mathrm{SiO}_{2}\right)\right]$ refractive indices. The optical thicknesses of $L$-layers are equal to a half of quarter of the wavelength $\lambda_{0}$ and the optical thicknesses of $H$-layer are equal to a quarter of the wavelength $\lambda_{0}$, where $\lambda_{0}$ is some fixed wavelength, which determines a position of an edge of filter transmission. The basic system (LHL) ${ }^{12}$ is the the standard construction of long-wave cut-off filters and the system $[1.08(\mathrm{LHL})]^{3}$ is the matched system, in which the optical thicknesses of layers differ from appropriate optical thicknesses of a basic system at 1.08 of time. Fig. 3 presents the spectral dependence of the reflection of the long-wave cut-off filter at the various angles of incidence $\theta$.

It is well seen, that difference in a reflection of polarized and unpolarized radiation are insignificant while the angle of incidence does not exceed $20-25^{\circ}$. As the angle of incidence increases, the reflection band shifts towards a blue part of the spectrum. As the angle of incidence increases, the width of the high reflection band and the reflection coefficient for $s$-polarizzed radiation increase, whereas those for the $p$-polarized component decrease. This results in a substantial distortion of the

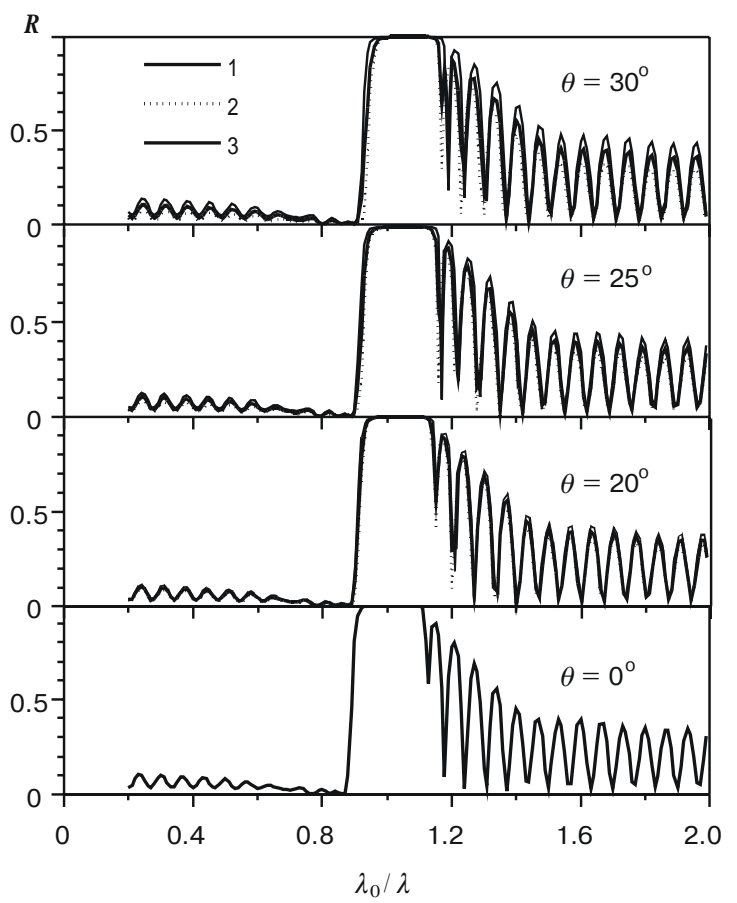

Fig. 2. Spectral dependences of reflection coefficients $R$ of the shotwave cut-off filter $\mathrm{S}(\mathrm{HLH})^{12}[0.92(\mathrm{HLH})]^{3}$ at various angles of incidence. The curve 1 corresponds to $s$-polarized radiation, 2 - to $p$-polarized radiation, and 3 - to unpolarized radiation. 


\section{V.Yu. Pervak et al.: Spectral properties of reflective interference filters}

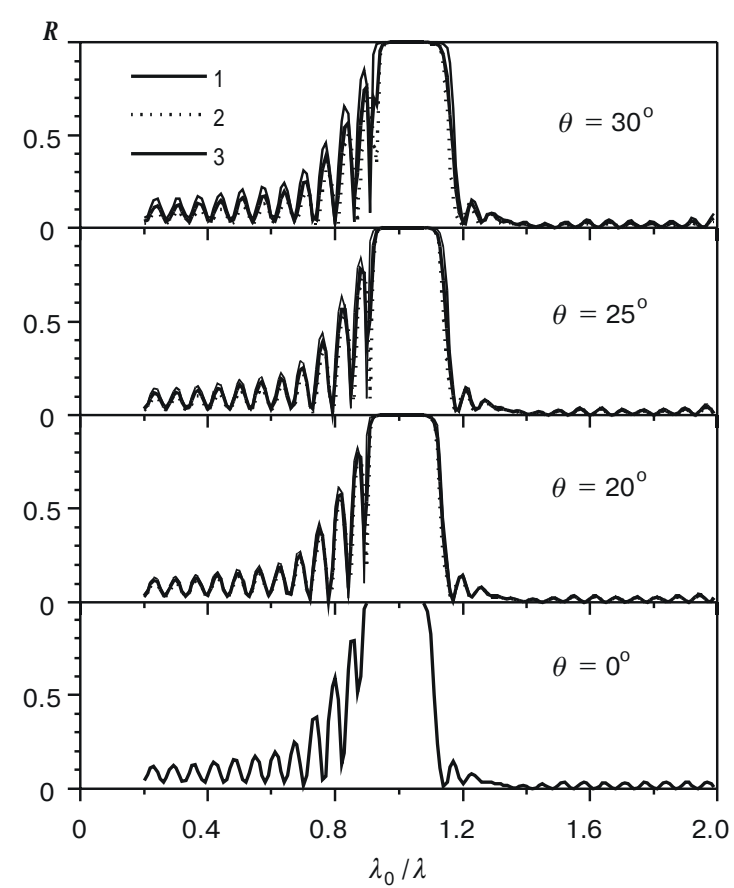

Fig. 3. Spectral dependences of reflection coefficients $R$ of the longwave cut-off filter $\mathrm{S}(\mathrm{LHL})^{12}[1.08(\mathrm{LHL})]^{3}$ at various angles of incidence. The curve 1 corresponds to $s$-polarized radiation, $2-$ to $p$-polarized radiation, and 3 - to unpolarized radiation.

reflection spectra of unpolarized radiation and in the decrease in the reflection coefficient.

Fig. 4 presents the spectral dependences of a transmission coefficient of a reflective filter, components of which are cut-off filters. In them there were 8 reflections when the angle of incidence on a surface of a filter is equal to $20^{\circ}$. It is necessary to mark that already at 4 reflections the transmission coefficient of a reflective filter in spectral region of a bandground is less then $10^{-4}$, at 6 reflections it is less then $10^{-5}$, and at 8 reflections it is less then $10^{-7}$, while in the field of a transparency it is close to unity (Fig. 5). At indicated parameters of multilayer systems the passband of a reflective filter is in limits $(0.9 \div 1.1) \lambda_{0}$ ( $\lambda$ ). In visible region of a spectrum at $\lambda_{0}=500 \mathrm{~nm}$ the reflective filter is transparent from $440 \mathrm{up}$ to $560 \mathrm{~nm}$. The half-width of a passband is equal to $100 \mathrm{~nm}$. Thus, in the spectral region from $450 \mathrm{up}$ to $540 \mathrm{~nm}$ the transmission is close to unity. The steepness of a shortwave edge of a passband is more than that of long wavelength. Thus the form of a passband almost varies with magnification of an amount of reflections inside a reflective filter. It is well seen in Fig. 6, where the spectral dependences of a reflective filter transmission for various numbers of reflections inside it and angle of incidence on reflecting surfaces equal $20^{\circ}$ are reduced. Besides at such angle of incidence the dependence of the form of a passband on polarization is insignificant. The shortwave edge of the passband is shifted by some nanometers to the longwave side and the longwave edge of the passband is shifted by some nanometers to the shotwave side for $p$-polarized radiation as compared with that of $s$-polarized.
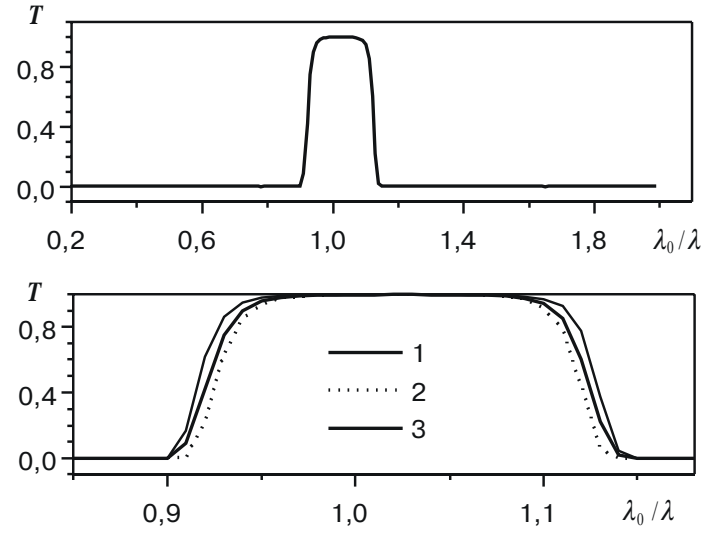

Fig.4. Spectral dependences of transmission coefficient $T$ of reflective filter, in which incident radiation till 4 times is reflected from surfaces of cut-off filters $\mathrm{S}(\mathrm{HLH})^{12}[0.92(\mathrm{HLH})]^{3}$ and $\mathrm{S}(\mathrm{LHL})^{12}[1.08(\mathrm{LHL})]^{3}$. The curves 1 correspond to the $s$-polarized radiation, 2 to the $p$-polarized radiation, and 3 to unpolarized radiation.
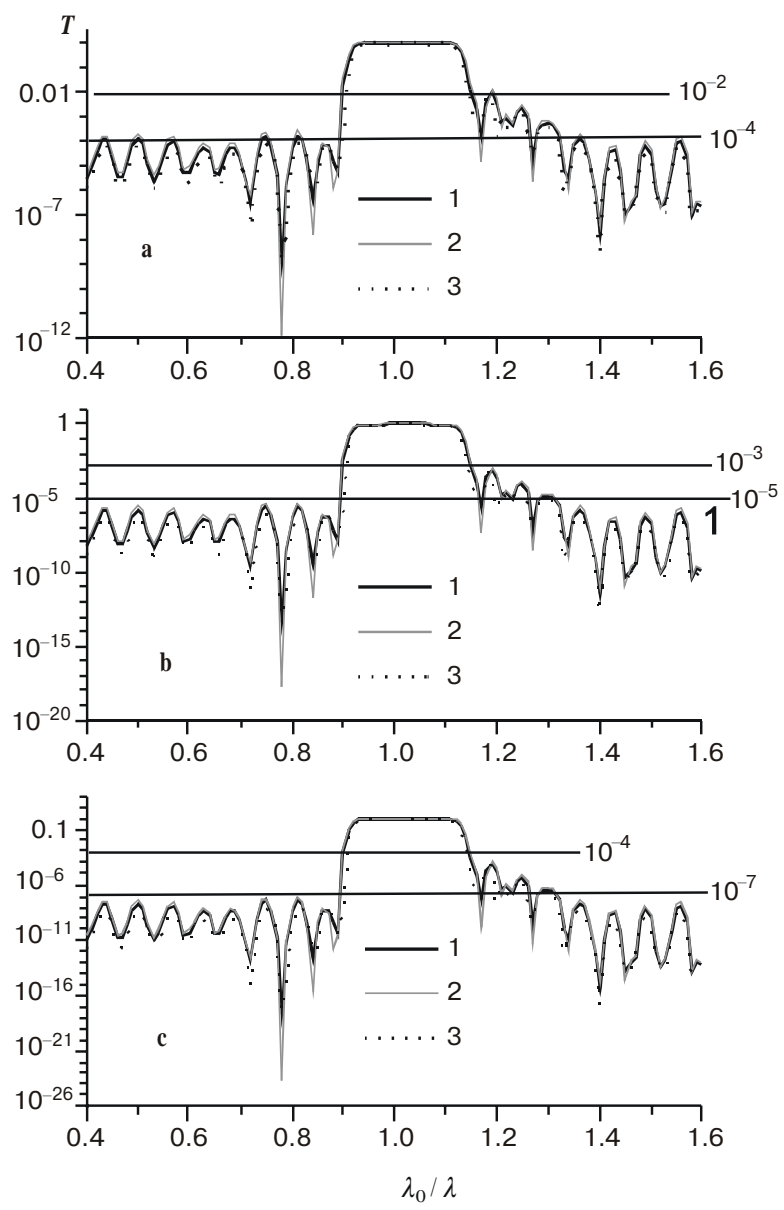

Fig. 5. Spectral dependences of transmission coefficient $T$ of the reflective filter, in which incident radiation up to 4 (a),6 (b) and 8 (c) times is reflected from surfaces of cut-off filters $\mathrm{S}(\mathrm{HLH})^{12}[0.92(\mathrm{HLH})]^{3}$ and $\mathrm{S}(\mathrm{LHL})^{12}[1.08(\mathrm{LHL})]^{3}$. The angle of incidence to a surface of the filter is equal to $20^{\circ}$. The curve 1 corresponds to $s$-polarized radiation, 2 - to $p$-polarized radiation, and 3 - to unpolarized radiation. 
V.Yu. Pervak et al.: Spectral properties of reflective interference filters

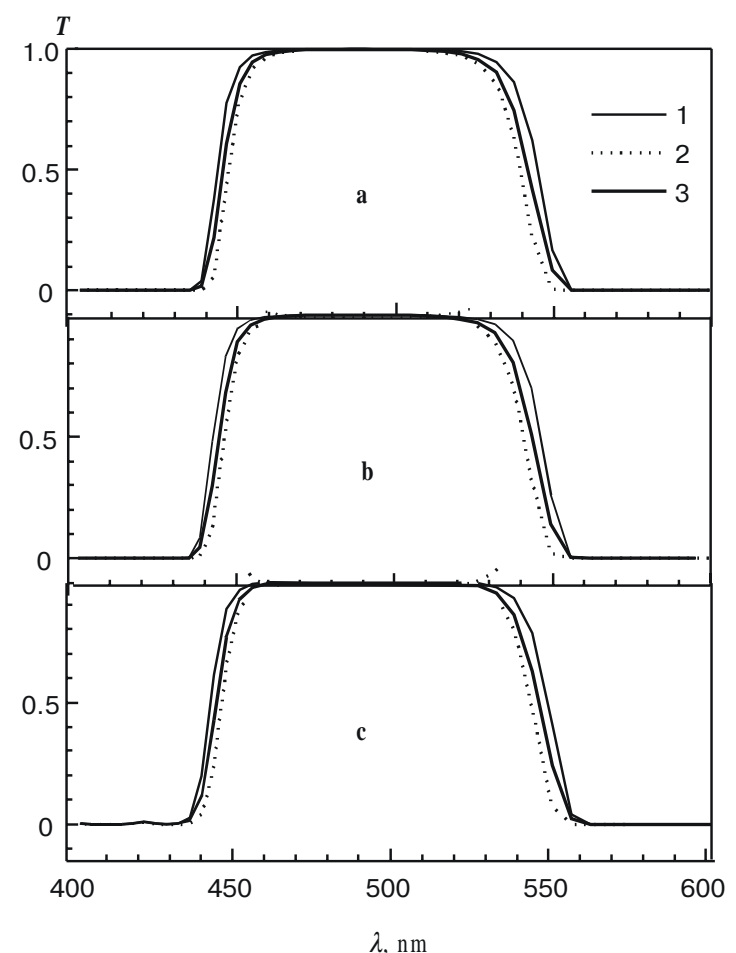

Fig. 6. Spectral dependences of transmission coefficient $T$ of reflective filter in which incident radiation up to 4 (a), 6 (b) and 8 (c) times is reflected from surfaces of cut-off filters $\mathrm{S}(\mathrm{HLH})^{12}[0.92(\mathrm{HLH})]^{3}$ and $\mathrm{S}(\mathrm{LHL})^{12}[1.08(\mathrm{LHL})]^{3} . \lambda_{0}=500 \mathrm{~nm}$. The angle of incidence to a surface of the filter is equal to $20^{\circ}$. The curve 1 corresponds to $s$-polarized radiation, 2 - to $p$-polarized radiation, and 3 - to unpolarized radiation.

It is possible to receive a reflective filter with a halfwidth of a passband $10-20 \mathrm{~nm}$. For this purpose it is necessary that superimpose region of suppression bands of the cut-off filters was as small as possible. It is obvious, that for reaching a minimum half-width of a reflective filter it is necessary to increase a steepness of passband edges of the cut-off filters. It becomes possible when multiplying the number of periods in a basic system of cut-off filters. However, substantial growth of number of layers, on the other hand, is not so desirable, because of the more complicated process of fabrication of coatings. As a result of optimization, the following constructions of cutoff filters were obtained. The shotwave cut-off filter consists of the fused silica substrate $S\left(n_{S}=1.45\right)$ and the multilayer system $(\mathrm{HLH})^{17}[0.92(\mathrm{HLH})]^{3}$ formed by the alternating layers with the high $\left[n_{H}=2.0\left(\mathrm{HfO}_{2}\right)\right]$ and low $\left[n_{L}=1.45\left(\mathrm{SiO}_{2}\right)\right]$ refractive indices. The optical thicknesses of $H$-layers are equal to half of quarter of the wavelength $\lambda_{0}$, and the optical thicknesses of $L$-layer are equal to a quarter of the wavelength $\lambda_{0}$, where $\lambda_{0}=430 \mathrm{~nm}$. The basic system (HLH) ${ }^{17}$ is the standard construction of shotwave cut-off filters and the system $[0.92(\mathrm{HLH})]^{3}$ is the matched system, in which the optical thicknesses of layers differ from appropriate optical thicknesses of the

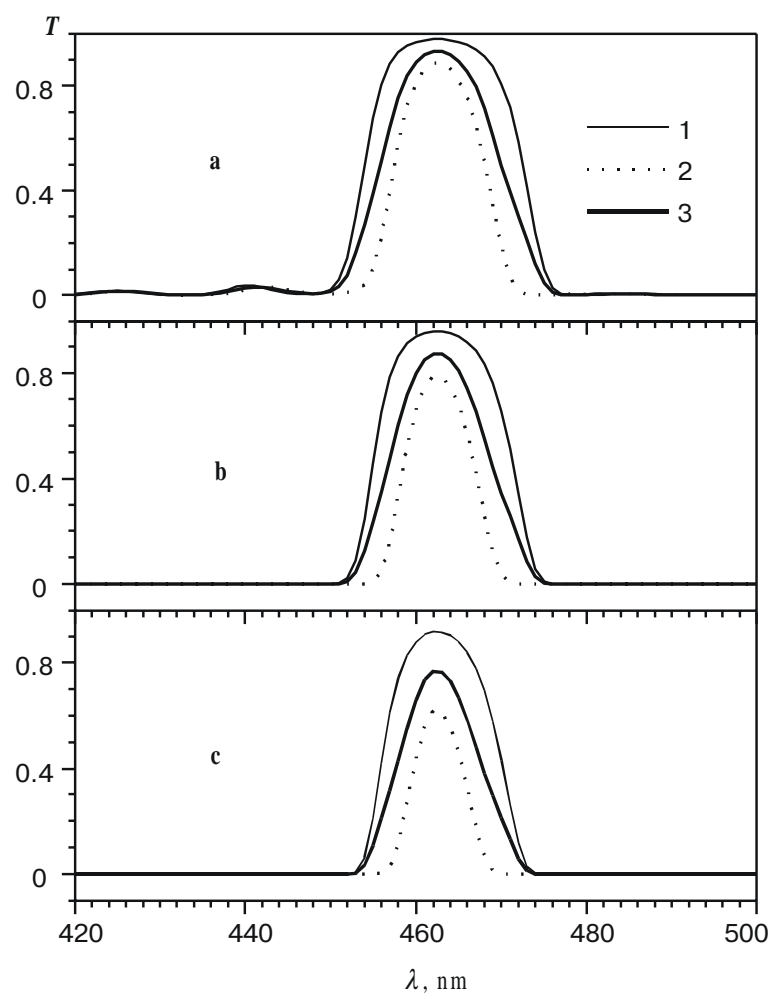

Fig. 7. Spectral dependences of transmission coefficient $T$ of reflective filter in which incident radiation up to 4 (a), 8 (b) and 16 (c) times is reflected from surfaces of cut-off filters $\mathrm{S}(\mathrm{HLH} 1)^{17}[0.92(\mathrm{HLH})]^{3}$ and $\mathrm{S}(\mathrm{LHL})^{17}[1.08(\mathrm{LHL})]^{3}$. The angle of incidence to a surface of the filter is equal to $20^{\circ}$. The curve 1 corresponds to $s$-polarized radiation, 2 - to $p$-polarized radiation, and 3 - to unpolarized radiation.

basic system by the factor of 0.92 . The longwave cut-off filter consists of the fused silica substrate $S\left(n_{S}=1.45\right)$ and the multilayer system (LHL) ${ }^{17}[1.08 \text { (LHL) }]^{3}$ formed by the alternating layers with the high $\left[n_{H}=2.0\left(\mathrm{HfO}_{2}\right)\right]$ and low $\left[n_{L}=1.45\left(\mathrm{SiO}_{2}\right)\right]$ refractive indices. The optical thicknesses of $L$-layers are equal to a half of quarter of the wavelength $\lambda_{0}$, and the optical thicknesses of $H$-layer are equal to a quarter of the wavelength $\lambda_{0}$, where $\lambda_{0}=500 \mathrm{~nm}$. The basic system (LHL) ${ }^{17}$ is the standard construction of longwave cut-off filters, and the system $[1.08(\mathrm{LHL})]^{3}$ is the matched system in which optical thicknesses of layers differ from appropriate optical thicknesses of the basic system by the factor of 1.08. Fig. 7 presents the spectral dependences of transmission coefficient $T$ of the reflective filter, in which incident radiation up to 4 (a), 8 (b) and 16 (c) times is reflected from surfaces of cut-off filters $\mathrm{S}(\mathrm{HLH} 1)^{17}[0.92(\mathrm{HLH})]^{3}$ and S(LHL) ${ }^{17}[1.08(\text { LHL })]^{3}$. A width of a passband and intensity of a passed radiation are decreased when an amount of reflections from cut-off filters increases. The most essential diminution is observed for $p$-polarized radiation. Intensity and width of a passband for $p$-polarized radiation considerably decrease at magnification of an angle of incidence. At the magnification of an angle of incidence the width of a passband 


\section{V.Yu. Pervak et al.: Spectral properties of reflective interference filters}
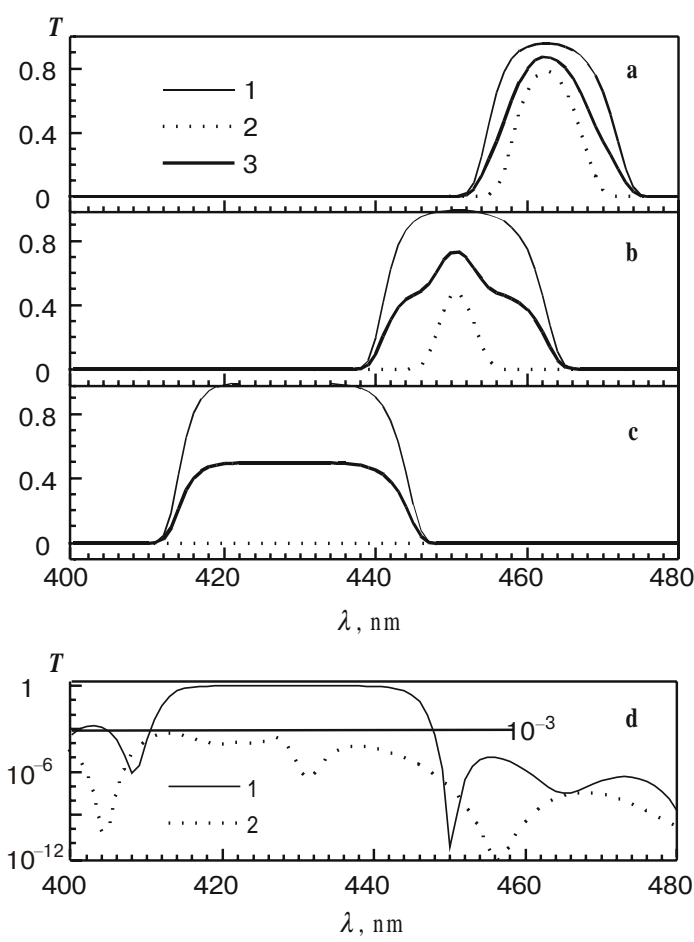

Fig. 8. Spectral dependences of transmission coefficient $T$ of reflective filter, in which incident radiation up to 8 times is reflected from surfaces of cut-off filters $\mathrm{S}(\mathrm{HLH}){ }^{17}[0.92(\mathrm{HLH})]^{3}$ and $\mathrm{S}(\mathrm{LHL})^{17}[1.08(\mathrm{LHL})]^{3}$. The angle of incidence to a surface of the filter is equal to $20^{\circ}$ (a), $30^{\circ}$ (b), $45^{\circ}$ (c, d). The curve 1 corresponds to $s$-polarized radiation, 2 - to $p$-polarized radiation, and 3 - to unpolarizzed radiation.

s-polarized radiation is increased, and that of p-polarized radiation is decreased. The filter transmits only spolarized radiation, when angle of incidence is equal to $45^{\circ}$. Fig. 8 presents the spectral dependences of transmission coefficient $T$ of the reflective filter, in which incident radiation is reflected up to 8 times between sur- faces of cut-off filters $\mathrm{S}(\mathrm{HLH})^{17}[0.92(\mathrm{HLH})]^{3}$ and $\mathrm{S}(\mathrm{LHL})^{17}[1.08(\mathrm{LHL})]^{3}$ at various angles of incidence. The magnification of an angle of incidence of a radiation from 20 up to $45^{\circ}$ resultes in the displacement of the filter passband center from $464 \mathrm{~nm}$ to $430 \mathrm{~nm}$. In the spectral range from 414 to $444 \mathrm{~nm}$ the researched reflective filter is an ideal polarizer, and the degree of polarization is close to $100 \%$.

The obtained results show that the use of reflective filters, which components are cut-off filters, allows considerably to expand functionalities of such multilayer interference systems. The more index of refractions of alternated layers in a structure of a filter will differ, the large breadth of a passband can be received, and it will be possible to reduce an amount of layers in interference coating. Besides such reflective bandpass filters are characterized by higher radiation damage threshold, as there are no components absorbing incident radiation in them.

\section{References}

1. V.Yu. Pervak, Yu.A. Pervak, Suppression of background transmission of interference filters // Opt.Spectrosc., 88 (3), pp. $455-458$ (2000).

2. Sh.A. Furman, Thin-Layer Optical Coatings. Mashinostroenie, Leningrad (1977).

3. H.A. McLeod, Thin-Film Optical Filters. Adam Hilger, London, (1985).

4. Yu.N. Markov, Synthesis of contrast band interference filters, Multihalfwave systems // Opt.Spectrosc., 53, pp. 537542 (1982).

5. Yu.N. Markov, Synthesis of contrast band interference filters, Complicatedly periodic systems // Opt.Spectrosc., 54, pp. 99-105 (1983).

6. P.P. Yakovlev, B.B. Meshkov, Design of Interference Coating. Mashinostroenie, Moscow (1987).

7. Yu.A.Pervak, I.V.Fekeshgazi, Optical properties of unequal thicknesses multilayer coatings with symmetrical periods // Quant. Electronic, Ukraine, No 46, pp. 8-11 (1994).

8. Yu.A. Pervak, I.V. Fekeshgazi, Optical properties of unequal thicknesses multilayer coatings with symmetrical periods at a sloping falling // Ukrainian Journal of Physics, 39 (6), pp. 668-672 (1994). 